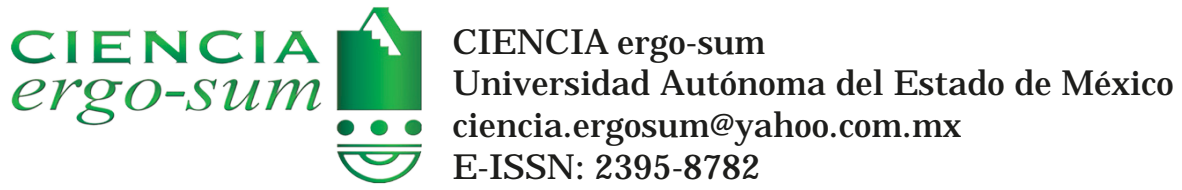

\title{
Soberanía alimentaria y tecnologías sociales: una experiencia de desarrollo autónomo desde los Andes del Perú
}

Ariza Arias, J ulian Andres; Morante Trigoso, Federico Bernardino

Soberanía alimentaria y tecnologías sociales: una experiencia de desarrollo autónomo desde los Andes del Perú

CIENCIA ergo-sum, vol. 26, núm. 2, julio-octubre 2019|e48

Universidad Autónoma del Estado de México, México

Esta obra está bajo una_Licencia Creative Commons Atribución-NoComercial-SinDerivar 4.0 Internacional.

Ariza Arias, J . A y M orante Trigoso, F. B. (2019). Soberanía al imentaria y tecnologías sociales: una experiencia de desarrollo autónomo desdelos Andes del Perú. CIE NCIA ergo-sum, 26(2). https://doi.org/10.30878/ces.v26n2a2 


\title{
Soberanía alimentaria y tecnologías sociales: una experiencia de desarrollo autónomo desde los Andes del Perú
}

Food sovereignty and social technologies: an autonomous development experience from the Andes of Peru

Julian Andres Ariza Arias

Universidade Federal do ABC, Brasil

julian.ariza.arias@gmail.com

Recepción: 04 de octubre de 2017

Aprobación: 03 de mayo de 2018

Federico Bernardino Morante Trigoso

Universidade Federal do ABC, Brasil

federico.trigoso@ufabc.edu.br

\section{RESUMEN}

La construcción de autonomía territorial en contextos rurales ofrece soluciones estructurales a la pobreza y a la marginalización de muchas comunidades en Latinoamérica. Además del acceso a tierra, la búsqueda de soberanía requiere capacidades organizativas y control de recursos y tecnologías. Desde esta visión, se presenta la propuesta de organización campesina de Sierra Productiva (SP) en el Perú y se generan algunas aproximaciones a una concepción integral de la soberanía. Se utilizó una metodología cualitativa basada en la observación participante y en entrevistas abiertas y semiestructuradas. Los principios de esta propuesta son coherentes con una visión del desarrollo a escala humana y de conquistas progresivas en la autonomía local. La experiencia de Sierra Productiva puede situarse como referencia regional de innovación en procesos comunitarios construidos "desde abajo".

PALABRAS CLAVE: organización, sierra productiva, yachachiq, agroecología, poder social.

\begin{abstract}
The construction of territorial autonomy in rural contexts can offer structural solutions to poverty and marginalization of many communities in Latin America. In addition to access to land, spaces of autonomy require organizational capacities and control of resources and technologies. This paper aims to present the proposed peasant organization of Sierra Productiva (SP) in Peru, generating some approximations to an integral conception of sovereignty. A qualitative methodology based on participant observation and open- and semi-structured interviews was used. The principles of this proposal are coherent with a vision of human scale development and progressive achievements in local autonomy. The SP process can be positioned as a regional reference for innovation in process of community empowerment built "from the bottom".
\end{abstract}

KEYWORDS: organization, profitable mountains, yachachiq, agroecology, social power.

\section{INTRODUCCIÓN}

Las apuestas comunitarias de organización nacidas "desde abajo" están orientadas por la construcción de prácticas democráticas directas que reconocen el protagonismo de las personas en su territorio. La satisfacción de necesidades, el desarrollo de capacidades endógenas para la organización y la incidencia en espacios de decisión son elementos característicos de procesos comunitarios que pueden configurar una respuesta sólida a las realidades rurales de exclusión y pobreza (Carpio y Elizalde, 2009; Portales, 2014). La escala local es la manifestación potencial de un espacio de autonomía que puede facilitar la satisfacción de necesidades y contribuir con un genuino proceso de desarrollo humano (Max-Neef et al., 2010).

Esas disputas por autonomía, que suceden en espacios geográficos concretos, estrechan el vínculo de las personas con el territorio al crear un conjunto de significados que alimentan la posibilidad de subvertir el orden instituido (Porto-Gonçalves, 2006). Con esta perspectiva se van construyendo territorialidades que reconocen las tensiones 
generadas por la defensa del espacio, en la medida que crean representaciones contrahegemónicas de los escenarios donde se juega la vida social. Estas territorialidades cuestionan la estructura colonial del proyecto de modernidad sobreponiendo a la noción establecida del desarrollo la necesidad de autonomía (Porto-Gonçalves, 2009). La lucha por establecer normas propias y recuperar el control del destino es una expresión de libertad que conjuga lo utópico, lo simbólico y lo posible con lo presente y lo tangible que también se manifiesta en el territorio.

La autonomía es uno de los pilares de una estrategia emancipadora que se concreta en los proyectos territorializados y se orienta a la construcción de poder social (Toledo, 2009). Esta estrategia se presenta como una alternativa a la crisis de la modernidad y asume una visión radical de la democracia que permitiría generar mecanismos para proteger a las comunidades de la economía global (Bajo Tierra, 2011; Baschet, 2015). Un elemento central para sustentar los espacios de autonomía, y que se deriva de los procesos históricos de organización comunitaria en América Latina, está asociado a la construcción de autosuficiencia alimentaria y la diversificación productiva (Barkin, 2002; Barkin y Lemus, 2015). La relación con el alimento permitiría afianzar los vínculos con el territorio, fortalecer lazos de solidaridad, resignificar saberes tradicionales y crear una estructura que ampliaría la condición de autonomía a otros dominios.

Actualmente, una de las apuestas sociales más amplias orientadas a la construcción de autonomía en los territorios, a partir de la transformación del sistema alimentario, se construye con el movimiento por la soberanía alimentaria (Rosset, 2016). El enfoque de la soberanía se sustenta en una visión de derechos, la cual reivindica algunos que inclusive no son reconocidos por instituciones internacionales. Además del derecho a la alimentación, se incluye el derecho a producir alimentos y al acceso a recursos para producirlos, el derecho a decidir lo que se quiere consumir y el derecho a decidir el nivel de autosuficiencia en los territorios (Heinisch, 2013; Rivera-Ferre, 2008).

La agroecología es uno de los pilares en la construcción de la soberanía alimentaria (Martinez-Torres y Rosset, 2014), ya que presenta una disputa por nuevas territorialidades orientadas a transformar las realidades rurales (Fernandes, 2017). La agroecología y la soberanía alimentaria, en tanto movimiento social, van más allá del acceso a los alimentos y la eliminación de dependencias energéticas en las unidades productivas. Ambas representan tanto un proyecto ecológico como una propuesta económica y social orientada a erradicar la pobreza y superar las relaciones sociales de opresión (Francis y Wezel, 2015; Heinisch, 2013).

La agroecología se presenta no sólo como un sistema alternativo de producción de alimentos sino como una estrategia sistémica de desarrollo rural. Ese proceso es orientado por la consolidación de la agroecología como enfoque científico y como expresión de saberes, conocimientos y experiencias propias de los(as) agricultores(as) (Caporal y Costabeber, 2004; Sevilla-Guzmán y Montiel, 2010). Además de convertirse en el fundamento de una estrategia de autonomía vinculada al territorio, la agroecología constituye la matriz energética y tecnológica de la soberanía alimentaria (Altieri y Toledo, 2011). Las soberanías energética y tecnológica también deben entenderse más allá del análisis de los agroecosistemas biodiversificados y asociarse con las condiciones necesarias para el acceso, la gestión y el control de los recursos. De esta manera, puede integrarse en la construcción de la soberanía el potencial libertador de la ciencia y la tecnología moderna para garantizar la permanencia y el bienestar de las comunidades en los territorios (Pimbert, 2009).

El acceso a estas herramientas tecnológicas reforzaría el papel de centralidad del mundo rural a través de la revalorización y modernización de las tradiciones productivas. Estas herramientas permitirían potenciar la participación, la expansión de las capacidades y el protagonismo de las personas al ampliar los espacios de libertad en las comunidades (Fernández-Baldor, 2014). También estimularían la organización de los movimientos locales para el acceso a tecnologías de vanguardia, la transferencia rural-rural y el desarrollo de nuevas herramientas en función de los saberes locales y los contextos de los(as) agricultores(as) (Carpio, 2002). Las tecnologías para la soberanía buscarían resolver los problemas estructurales de productividad, infraestructura y acceso a recursos; además, presentarían un componente político que las oriente a los grupos históricamente marginalizados (CIPAF-INTA, 2009). 
Los esfuerzos para entender las soberanías como elementos integrados y las prácticas en el territorio como la proyección de un espacio tecnológico coherente deben constituir una pauta central en los procesos de construcción de autonomía aceptando que las tecnologías inducen procesos de cambio social, ya que condicionan estructuras de distribución y acceso a bienes y servicios, generan o resuelven conflictos socioambientales y promueven condiciones de exclusión o inclusión social (Thomas, 2011). Un conjunto de herramientas al servicio y bajo el control de las comunidades fortalece las construcciones de identidad, los valores colectivos, los imaginarios de futuro, las perspectivas de autonomía y las lecturas críticas de la realidad (Illich, 1978).

A partir de lo expuesto, el objetivo del artículo es presentar la propuesta de organización campesina de Sierra Productiva (SP) en el Perú por medio de la búsqueda de articulaciones con una visión integral de la soberanía. Sierra Productiva es una iniciativa de desarrollo rural que surge de un proceso histórico de lucha campesina protagonizado en la región de Cusco. Se realizó una investigación cualitativa de carácter descriptivo/exploratorio mediante observación participante y desarrollo de entrevistas abiertas y semiestructuradas con participantes clave (Guber, 2011; Sampieri et al., 2010). Con estas herramientas, y con el apoyo en documentación interna del Instituto para una Alternativa Agraria (IAA), se generó una aproximación al ámbito cultural y social de las comunidades. La visita de campo fue hecha entre el 21 y el 27 de julio de 2015. Las entrevistas abiertas fueron desarrolladas con campesinos y campesinas pertenecientes a las regiones de Canas, Paucartambo, Calca y Acomayo, en la región Cusco. Estas entrevistas contaron con el acompañamiento del IAA, que hizo las veces de mediador y se encargó de facilitar los contactos, así como el acercamiento con las personas. La entrevista semiestructurada se realizó en la ciudad de Cusco con la directora ejecutiva del IAA y con el coordinador nacional de Sierra Productiva.

En la segunda parte del trabajo se presenta un breve recuento histórico de los procesos de lucha por la tierra en Perú. En la tercera, se muestra el proceso de organización y consolidación de la propuesta de Sierra Productiva a partir de los relatos de algunos(as) campesinos(as) y una contextualización documental. Por último, se plantean las consideraciones finales destacando algunos de los elementos que pueden situar la experiencia como una referencia legítima de construcción de autonomía y se proponen perspectivas futuras de investigación.

\section{Lucha por la tierra en Perú}

La tragedia histórica de las comunidades campesinas e indígenas en Perú se remonta a la invasión del imperio inca por parte de los españoles en 1532. Con la destrucción de la organización incaica, se instauró una herencia colonial caracterizada por un régimen económico feudal con elementos esclavistas que tenía como expresiones el latifundio y la servidumbre (Mariátegui, 1928/1986).

Según Villanueva (1967) hasta antes de la primera reforma agraria protagonizada en Cusco en 1962, las condiciones de vida de la mayoría de campesinos(as) eran deplorables. El hacendado o gamonal, usando su conocimiento de lenguas (quechua o aymara y español), estableció un dominio inhumano sobre el campesinado analfabeto. Esto alimentó las luchas populares en la provincia de La Convención, las cuales pasaron por la implementación de reformas agrarias radicales mediante la apropiación de tierras y la expulsión de los gamonales. A pesar de su ilegalidad, las manifestaciones fueron exitosas y llevaron a la propagación de sindicatos y federaciones campesinas en la región (Villanueva, 1967).

Fue en este contexto de lucha por la reforma agraria que nació la Federación Departamental de Campesinos del Cusco (FDCC), que se convertiría en referente de organización campesina. Como resultado de la presión iniciada en los valles de La Convención y Lares, en 1962 fue promulgada por la Junta Militar de gobierno una ley de reforma agraria que en la práctica validaba las ocupaciones de tierras en Cusco (Cotler y Portocarrero, 1976). Esta reforma agraria marginal representó una estrategia para apaciguar los conflictos sin mostrar soluciones estructurales para los campesinos. 
En 1964 el gobierno aprobó una ley de reforma agraria de ámbito nacional, pero que fue tímidamente aplicada. Para 1969 fue ejecutada una nueva ley de reforma agraria, esta vez con el apoyo de las fuerzas armadas lideradas por el general Juan Velasco Alvarado. Esta última reforma eliminó de manera formal el poder gamonal a partir de la expropiación total de los latifundios (Eguren, 2006). Sin embargo, al finalizar el periodo de implementación de la reforma sólo una cuarta parte de la población rural obtuvo acceso a la tierra (Rosas, 2009).

Esta reforma agraria que constituía el proyecto de cambio más importante de la época, al enfrentar el capitalismo agrario, se vio debilitada por las contradicciones internas y las enraizadas estructuras burocráticas (Matos Mar y Mejía, 1980). La decepción popular generada con la fallida implementación de las reformas contribuyó con el surgimiento de Sendero Luminoso. En 1970 este movimiento intensificó el trabajo ideológico en las áreas rurales como preludio de la insurgencia armada que aparecería en 1980 (Rénique, 2009).

Posteriormente, el gobierno de Morales Bermúdez (1975-1980) eliminó los programas desarrollados por Velasco y declaró de manera oficial el final de la reforma agraria. En el gobierno de Belaúnde Terry (1980-1985) se instauraron las políticas neoliberales provenientes de programas de ajuste estructural del Fondo Monetario Internacional y el Banco Mundial. En el primer gobierno de García Pérez (1985-1990) se intentó transformar la profunda marginalización del país a partir de la confrontación al capital internacional; no obstante, en esos años se profundizó la crisis social y la influencia de Sendero Luminoso (Rosas, 2009). Durante el gobierno de Alberto Fujimori (1990-2000) se consolidaron las medidas neoliberales, la corrupción consumió las instituciones y el Perú enfrentó la mayor crisis de su historia (Matos-Mar, 2005). Fue en este último periodo que nació la propuesta de desarrollo rural de Sierra Productiva a partir de la articulación de la FDCC y la Organización de la Sociedad Civil IAA.

\section{El nacimiento de Sierra Productiva}

Ante la ineficiencia de las manifestaciones populares de ese momento, la FDCC y el IAA iniciaron un proceso de reflexión que tuvo como elemento central la discusión de cómo producir sin la ayuda del estado. Las demandas de las comunidades habían pasado de la histórica lucha por la propiedad de la tierra a nuevas reivindicaciones como el acceso y la gestión de los recursos naturales, el uso eficiente del agua, la búsqueda de soluciones para las dificultades de la baja productividad, el acceso a mercados y la tecnificación de las pequeñas unidades familiares.

El estudio de formas de producción autónomas llevó a identificar en las prácticas agroecológicas y en las tecnologías apropiadas las bases para la construcción de una alternativa de desarrollo endógena. Fue así que en 1994 nació la propuesta de Sierra Productiva, la cual asumió una visión territorial del desarrollo y se respaldó en un proceso educativo de campesino a campesino liderado por los yachachiq. Esta palabra quechua, que significa "aquel que tiene el conocimiento y hace que otro aprenda", refiere a un campesino o campesina que se destaca en la comunidad por su creatividad y liderazgo. Históricamente, el yachachiq ha sido la persona que practica el ayni y la minka, prácticas de reciprocidad y trabajo colectivo tradicionales del mundo andino (Vivanco y Bellatín, 2013).

El ejercicio educativo se articuló con un plan metodológico conocido como la escalera del progreso (figura 1). La escalera marcó las fases de implementación de tecnologías y representó un camino guía para la superación de la pobreza en las comunidades. Las etapas de la escalera están conformadas por a) capacitación básica y réplicas, b) instalación de tecnologías de costo cero, $c$ ) adecuación de infraestructura productiva, $d$ ) mejoramiento del ganado y las semillas, $e$ ) implementación de tecnologías apropiadas con uso de energías renovables, $f$ ) implementación de módulos de transformación familiar, $g$ ) transformación asociada de productos, $h$ ) desarrollo integral de la microcuenca, $i$ ) planes de producción y comercialización. 


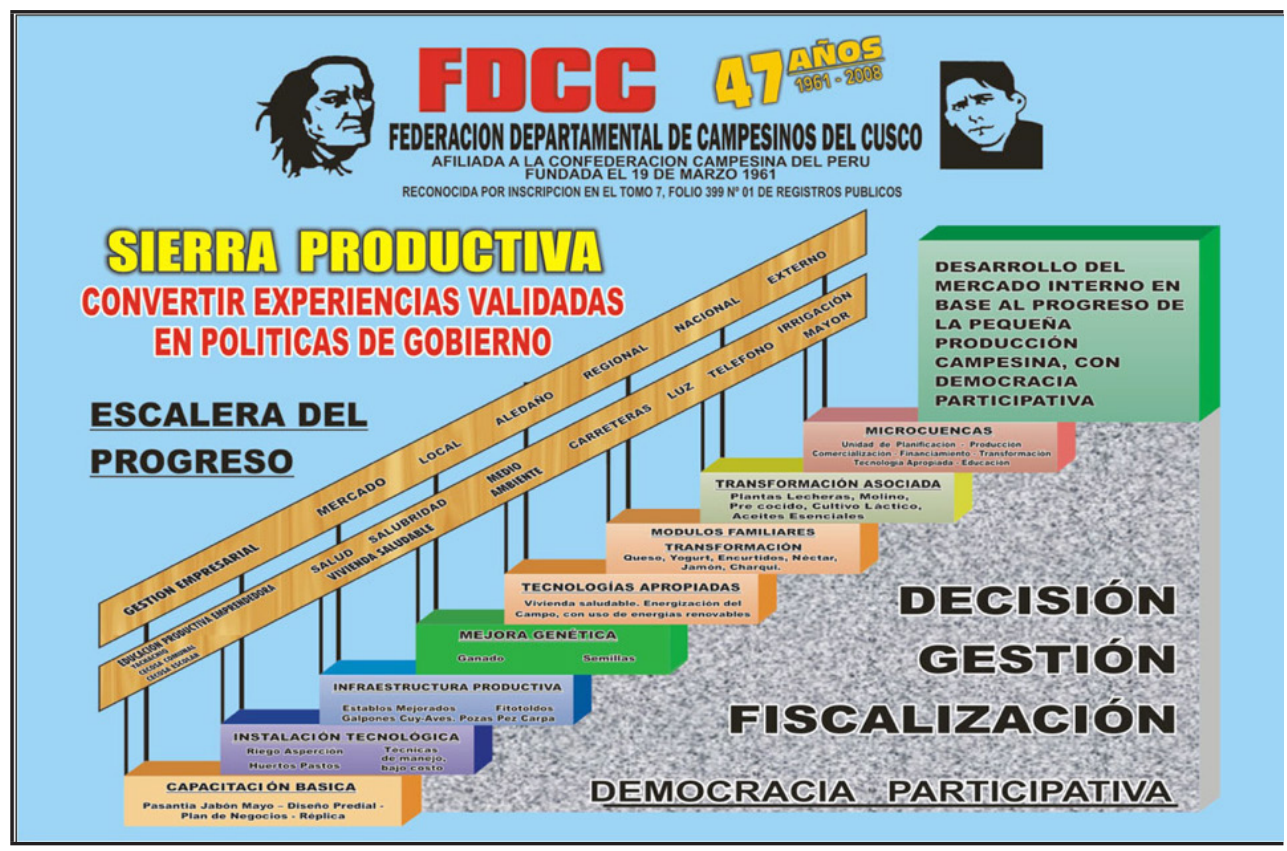

FIGURA 1

La escalera del progreso

Fuente: documentos internos IAA.

En palabras de uno de los yachachiq que acompañó el proceso de formación de Sierra Productiva:

Nuestra escalera del progreso es simple. Usted ve y aprende. Usted va a encontrar en el primer escalón la irrigación, entonces, teniendo irrigación usted puede sembrar pasto, teniendo pasto usted puede tener ganado, con ganado usted puede obtener leche, con la leche usted puede producir yogur o queso. Hay una secuencia y lo mismo pasa con las hortalizas (entrevistado 1, entrevista personal, 25 de julio de 2015).

El soporte de la escalera del progreso es la democracia participativa conquistada gradualmente con las luchas campesinas. Con el acceso a la tierra en algunas regiones, el interés de los movimientos campesinos pasó a la apertura de espacios de diálogo con autoridades. En particular, fueron importantes los esfuerzos orientados a realizar la elección de alcaldes mediante voto popular, los esfuerzos de mujeres campesinas para cogestionar programas sociales de gobierno y la institucionalización de asambleas campesinas en la región de Limatambo.

Estas asambleas ganaron prestigio en las comunidades y después, bajo el nombre de Consejo Comunal Vecinal, fueron avanzando hasta configurar la garantía de tres derechos: a) derecho a participar en la toma de decisiones sobre temas de interés público, plan estratégico de desarrollo y presupuestos, $b$ ) derecho a participar en la gestión para garantizar el cumplimiento de los acuerdos y $c$ ) derecho a participar en la fiscalización social sobre el uso del dinero y los comportamientos de las autoridades.

La primera implementación del modelo de Sierra Productiva se dio en 1994 en la microcuenca del Jabón Mayo, localizada en la provincia de Canas, Cusco. Esta región, ubicada entre los 3700 y $4800 \mathrm{msnm}$ se caracterizaba por las dificultades de acceso a agua y tecnología, la limitada producción agrícola de subsistencia y los elevados índices de desnutrición principalmente en niños. Esa primera experiencia fue llamada "Alternativa integral de desarrollo local con base al progreso de la pequeña producción campesina” y tuvo como objetivos: a) el desarrollo de habilidades mediante capacitación tecnológica para garantizar seguridad alimentaria e incremento de renta en las familias y b) la promoción de una agroindustria artesanal para la creación de valor agregado y la generación de ganancia. 
La estrategia estuvo acompañada de un plan de gestión de la microcuenca que permitió cambiar el sistema de riego en las comunidades. Se introdujo el riego tecnificado y fueron desarrollados varios tipos de aspersores artesanales, adaptados a las particularidades de cada unidad familiar. Junto con la adopción del riego, también se inició la capacitación para hacer la transición productiva hacia un modelo agroecológico. La transmisión de conocimientos estuvo mediada por los yachachiq que participaban en actividades de capacitación facilitadas por el IAA y después compartían los conocimientos en sus comunidades. Ese proceso era realizado en quechua y con la metodología del "aprender haciendo".

Para ese momento, se construyeron en las comunidades y escuelas los Centros Comunales de Seguridad Alimentaria (CECOSA) que servían como centros de experimentación y transferencia de conocimientos. Posteriormente, por iniciativa de la comunidad fueron creados los Centros Familiares de Seguridad Alimentaria (CEFASA) con el objetivo de que las familias replicaran en sus casas todo lo que habían aprendido. Sobre estos centros un miembro de la FDCC comenta:

En esos CECOSA aprendí muchas cosas, más de cinco años investigando y practicando [...] todos los comuneros hombres y mujeres tenían que ir los sábados a la capacitación: todo sobre cómo sembrar el pasto, cómo preparar los terrenos, cómo preparar compost orgánico, cómo hacer invernaderos, cómo hacer crianza de cuyes y aves, cómo podíamos transformar los productos (entrevistado 2, entrevista personal, 25 de julio de 2015)

Las tecnologías implementadas permitieron a las familias pasar de una alimentación precaria y dependiente a una diversificada, nutritiva y con un alto grado de autosuficiencia. Gracias a los sistemas de riego, a la adaptación de invernaderos a más de $4800 \mathrm{msnm}$, al cultivo de pastos asociados, a las plantaciones escalonadas, a la rotación de cultivos, al uso de biofertilizantes y abonos orgánicos, control biológico de plagas, entre otros, se consiguió cultivar hasta 30 variedades de hortalizas, frutas tropicales, variedades de granos, tubérculos y plantas aromáticas y medicinales (Paredes, 2008). Ese proceso también fortaleció prácticas como el almacenamiento de alimentos para garantizar la disponibilidad de comida ante eventuales crisis. Como lo señala una yachachiq y promotora de salud de las comunidades:

Tengo una despensa llena de alimentos. Siempre hay reservas de comida de años. De repente este año no hay nada, llega la helada o el granizo y no hay maíz. De repente en dos o tres años no va a haber, por eso guardamos (entrevistada 3, entrevista personal, 24 de julio de 2015).

El florecimiento productivo en las pequeñas unidades familiares permitió el aumento en los volúmenes de la cosecha y la diversificación de los cultivos. El proceso de educación no escolarizada y entre iguales promovió un cambio de actitud en la comunidad, mejoró significativamente la autopercepción y fortaleció las organizaciones locales.

\section{LA CONSOLIDACIÓN}

A partir del año 2000 fue formado un equipo nacional de yachachiq que facilitó la difusión de la propuesta en otras regiones del Perú. En 2002 se formalizó la estrategia de transición agroecológica basada en el rescate de los conocimientos andinos, en la gestión integral de las microcuencas y en el desarrollo de capacidades en las comunidades para la resolución de sus propios problemas. Para 2004, se inició un proceso de transferencia tecnológica facilitado por la Pontificia Universidad Católica del Perú (PUCP). Este convenio fue consolidado por el interés de Sierra Productiva de tecnificar la pequeña producción campesina. De esta forma, fue elaborado el proyecto "Capacitación para la Tecnificación y Energización del Agro de la Pequeña Producción Campesina". El proyecto hizo parte del programa de desarrollo local con democracia participativa y contó con el apoyo de la Cooperación Española. 
El objetivo fue "capacitar capacitadores" campesinos(as) (yachachiq) en el manejo, montaje y construcción de equipos basados en energías renovables. Inicialmente, se acudió a los congresos campesinos para presentar algunas tecnologías que ya habían sido validadas por el Grupo de Apoyo al Sector Rural y el Grupo de Métodos Computacionales de la PUCP. La difusión buscó motivar a los(as) campesinos(as) para realizar las capacitaciones en la fabricación, mantenimiento y uso de equipos agrícolas, así como en la atención de servicios básicos y en temas de desarrollo de la pequeña producción campesina. Esta tarea también se realizó en algunas instituciones educativas. Sobre esta experiencia, uno de los jóvenes yachachiq de energías renovables comenta:

En 2004 entramos a trabajar un convenio con la PUCP en el área de energías renovables. Seis estudiantes venimos a capacitarnos en Yanaoca. Participamos por un periodo de dos años seguidos. Todo lo aprendido de a poco fue llevado al colegio y se capacitó a padres, profesores y estudiantes. Fue un esfuerzo enorme. Estábamos quince días en la institución y quince días en las capacitaciones durante los últimos dos años de colegio (entrevistado 4, entrevista personal, 25 de julio de 2015).

En las capacitaciones fue usado un establecimiento fijo en la localidad de Yanaoca llamado yachaywasi, que en quechua significa "casa del saber", y que funcionó como centro práctico de estudio. La transmisión de conocimientos del yachaywasi al colegio generó innovaciones educativas que llamaron la atención en la región. Esta experiencia motivó el cambio del programa académico y la metodología de enseñanza de algunos cursos. Fueron integrados en la formación de algunas instituciones temas de nutrición, energías renovables y construcción de equipos como secadores y deshidratadores solares. Sobre estas innovaciones el yachachiq de energías renovables menciona:

Normalmente usted tomaba las matemáticas en cuatro paredes y fórmulas y cosas, pero de repente salimos al campo a trabajar las matemáticas con las plantas, con lo que se hacía en el campo. Y se entendió una forma muy distinta de aprender. Igual con la química; por ejemplo, cuando instalamos el biodigestor, ¿qué pasa con el gas? (entrevistado 4, entrevista personal, 25 de julio de 2015).

En el primer año del convenio con la PUCP fueron capacitados 65 yachachiq en tecnologías de bombeo de agua y generación de calor (cocinas solares, cocinas mejoradas, colectores solares y secadores de granos). Entre 2006 y 2007 los yachachiq capacitaron 986 familias en la fabricación, instalación y uso de colectores solares, estufas solares, secadores solares y sistemas de bombeo de agua. En algunas comunidades fueron instaladas ruedas hidráulicas y bombas de ariete, lo cual mejoró la irrigación y amplió la frontera agrícola.

Los yachachiq conocieron el funcionamiento de equipos para la generación de energía eléctrica como aerogeneradores y sistemas fotovoltaicos. En asociación con la Universidad Politécnica de Cataluña también conocieron la tecnología de biodigestión para la generación de biogas y biofertilizante. Los yachachiq continuaron difundiendo los conocimientos a otras comunidades de las regiones de Ayacucho, Apurímac, Arequipa, Huancavelica y Puno. La práctica del ayni en el proceso educativo mediado por los yachachiq fortaleció un compromiso solidario con las familias campesinas que permitió la realización de las tareas educativas sin ningún tipo de mediación económica. Sobre este ejercicio uno de los campesinos educadores comenta:

Hemos tenido la costumbre de los incas que es el ayni o minka. Yo voy a su casa y usted me corresponde con el mismo trabajo cuando yo esté necesitando. No se paga dinero (entrevistado 5, entrevista personal, 24 de julio de 2015).

Las experiencias del convenio fueron socializadas en otras 15 regiones del Perú y motivaron la incidencia en políticas de gobierno en 104 alcaldías del país. Este proceso ganó prestigio tanto en las ONG de cooperación para el desarrollo como en las instituciones públicas del Perú. En palabras de una evaluadora externa del proyecto:

El proyecto ha tenido un efecto multiplicador extraordinario, difícilmente encontrado en otros proyectos de desarrollo [...]. La experiencia debería ser analizada por especialistas en pedagogía, en metodologías educativas, en capacitación laboral técnica y en investigación científica y tecnológica para extraer aprendizajes a favor de una reforma educativa universitaria y escolar al servicio de las mejoras productivas y de calidad de vida en las comunidades (Velarde, documentos internos IAA, 2006: 17-24). 
Una vez terminado el proyecto, algunas tecnologías apropiadas se incorporaron en las comunidades para responder de forma integral al mejoramiento de las condiciones de vida. Entre esas tecnologías se encontraban diversos tipos de bombas de agua, aspersores artesanales, sistemas fotovoltaicos, aerogeneradores eléctricos, secadoras y deshidratadoras solares, cocinas solares, termas solares, baños secos y biodigestores (Morante, 2007). Después de llevar la propuesta de Sierra Productiva a otras regiones y de probar más de 40 opciones tecnológicas, fue consolidado un conjunto de 18 tecnologías clasificadas en productivas y de mejoramiento de los servicios básicos. Los resultados de la implementación de Sierra Productiva mostraron que la pequeña producción familiar es viable y que es posible eliminar la pobreza y la desnutrición mediante la ampliación del poder comunitario y el uso de tecnologías simples y baratas.

El fortalecimiento comunitario permitió la incidencia en políticas públicas para llevar Sierra Productiva a más comunidades. Este proceso pasó por el reconocimiento de malos manejos en algunas administraciones locales, dirigidas por alcaldes de otras regiones, que desviaban los recursos de presupuestos participativos y de fondos colectivos destinados a la comunidad. Sobre esta situación un dirigente de la FDCC señala:

La FDCC inició un trabajo político bajo el lema "campesino al poder". Desde la Federación dijimos: "sólo nosotros vamos a hacer el cambio". Entonces, trabajamos en la parte política y social y 2007 fue el primer año en que llevamos un alcalde del campo. Como nuestro líder llegó al poder, entonces, propusimos que Sierra Productiva participara en el municipio a través de los presupuestos nacionales (entrevistado 2, entrevista personal, 25 de julio de 2015).

Para facilitar la participación de recursos públicos en la expansión de la propuesta, se propuso que el conjunto de tecnologías fuera implementado en las comunidades en un periodo de tres años. Las tecnologías del primer año son riego por aspersión, huerto fijo a campo abierto, parcela de pastos asociados cultivados, módulo de crianza de cuyes, módulo de crianza de gallinas, producción de granos y tubérculos andinos, abonos orgánicos, agroflorestas, cocina mejorada y purificación de agua. En el segundo año se trabaja con infraestructura para el ganado, huerta en invernadero, módulo para la transformación artesanal, baño seco y colectores solares. Finalmente, las tecnologías implementadas en el tercer año son biodigestor, cocina solar y crianza de peces carpa.

La visión de Sierra Productiva es que 100\% de las familias del campo tenga biodigestores y que se consolide el uso de aerogeneradores, sistemas fotovoltaicos y pequeños aprovechamientos hidroeléctricos para generar energía en las comunidades aisladas. Además de las 18 tecnologías, también son instalados biosistemas integrados comunitarios para el tratamiento de aguas negras. Hacia 2014 Sierra Productiva inició un proceso de capacitación productiva con tecnologías de información y comunicación orientado a que las familias campesinas puedan estudiar desde sus casas y pongan en práctica los aprendizajes en sus unidades familiares.

La propuesta endógena de Sierra Productiva tiene cinco enfoques, construidos en respuesta a la visión mecanicista del desarrollo rural. Estos son:

a) Gestión integral de microcuencas: basada en principios agroecológicos. Es la esencia de la gestión territorial, en la cual el agua es el factor central para la delimitación del territorio.

b) Altas productividades en pequeños espacios: también basada en principios agroecológicos. Son usadas tecnologías apropiadas para diferentes condiciones ambientales.

c) Capacitación de campesino a campesino: basada en la democracia participativa y directa. Es el eje central de la transferencia de conocimientos desarrollado mediante la educación intercultural y no escolarizada mediada por los yachachiq.

d) Incidencia política: basada en la democracia participativa y directa. Surge del interés de los yachachiq y las familias beneficiarias por ampliar la propuesta a otras familias, comunidades, distritos y departamentos.

e) Construcción de mercado local y gestión empresarial: busca crear un dialogo entre los pequeños productores y los consumidores con el objetivo de desarrollar mercados locales justos y promover la industria de pequeña escala en las comunidades. 
Estos enfoques sustentan los cuatro componentes actuales de la propuesta: a) seguridad y soberanía alimentaria, b) generación de renta, empleo y autoempleo, c) educación productiva, emprendedora e innovadora y d) democracia participativa e incidencia en políticas. Durante la primera fase de implementación de Sierra Productiva, que fue entre 1994 y 1999, se beneficiaron 1800 familias. Posteriormente, con la expansión a nivel nacional entre 2000 y 2014, el programa llegó a 60000 familias. En la actualidad la propuesta es reproducida en comunidades pertenecientes a 16 de las 24 regiones del país. En las palabras de uno de los yachachiq, se refleja el grado de transformación generado por la propuesta de Sierra Productiva:

Hace cinco años que yo no visito el mercado para nada. Mis hijos viven en Lima y ahora quieren regresar. Ha cambiado la casa. Gracias a Sierra Productiva hemos mejorado la vivienda y todo mi ambiente. Estas casas son ejemplo para toda la comunidad de que el campesino puede vivir así. Esto ha cambiado bastante con las propuestas de Sierra Productiva. El campesino toda la vida ha sido odiado por los hacendados, por todos, el campesino no está atendido en oficinas públicas [...]. Ahora si recién el campesino está tomando valor, el campesino tiene su huerta familiar, tiene una vivienda, ya se siente como gente. Esas cosas han cambiado y estoy alegre con el cambio que hemos hecho (entrevistado 5, entrevista personal, 24 de julio de 2015).

Sierra Productiva ha ganado diferentes reconocimientos que tienen un significado especial por el hecho de ser un proceso que surge de la organización de comunidades campesinas y la sociedad civil. El inusitado respaldo se refleja, por ejemplo, en la conformación del Grupo de Apoyo al Programa Sierra Productiva, por parte de líderes de opinión, empresarios, investigadores y personalidades peruanas, con el objetivo de proteger la propuesta y evitar intromisiones que la distorsionen. Entre otros reconocimientos de Sierra Productiva, está la medalla de oro de la FAO (Food and Agriculture Organization) en el Día Mundial de la Alimentación en 2005, premio del Consejo Nacional de Ambiente Peruano (CONAM) en 2007, implementación de la propuesta como política pública a través del programa de gobierno Mi Chacra Productiva en 2009, consultoría del proyecto de mejoramiento de la nutrición en poblaciones indígenas de la Comunidad Andina de Naciones (CAN) en 2009, segundo lugar en un concurso internacional sobre programas de desarrollo e innovación social convocado por la empresa Shell, la revista Newsweek y la BBC de Londres en 2011 y el reconocimiento especial del vicedirector del Fondo Monetario Internacional, quien visitó algunas organizaciones en 2014 y destacó la importancia de la propuesta para extenderla a otros países.

\section{Consideraciones finales}

Este artículo buscó hacer una aproximación conceptual a la propuesta de Sierra Productiva en el marco de una visión del desarrollo rural asociada a la construcción de autonomía territorial y la soberanía integral. Una evaluación rigurosa de la propuesta supera los objetivos y el alcance de esta investigación. No obstante, es pertinente mostrar algunas apreciaciones generales construidas en función de las características del desarrollo a escala humana (Max-Neef et al., 2010) y de algunos principios de la soberanía alimentaria. La propuesta de Sierra Productiva está orientada a la satisfacción de las necesidades humanas teniendo como objetivo inicial atender el desafío de mejorar la alimentación en las comunidades. El proceso para alcanzarlo posibilitó una dinámica sinérgica de satisfacción de otras necesidades humanas como protección, participación, creatividad y libertad. Esto estuvo asociado a las capacidades construidas en relación con la autonomía, la organización y la solidaridad, entre otras.

La estrategia de Sierra Productiva surgió a partir de la pregunta de cómo producir sin la ayuda del estado. Con el progresivo fortalecimiento de la organización campesina y la validación de las tecnologías, las comunidades redujeron las dependencias externas mejorando sus condiciones de vida. Esto se evidencia, por ejemplo, cuando las familias pasan de subsistir por medio de la venta de su fuerza de trabajo a una condición de elevada autosuficiencia alimentaria en sus terrenos. En esa autosuficiencia se incluye la eliminación de los fertilizantes químicos y pesticidas, ya que las familias fabrican biofertilizantes y hacen un control biológico de plagas. Otra manifestación de los niveles de independencia se encuentra en el proceso educativo de campesino a campesino que les permite eliminar la permanencia de actores externos a las comunidades. 
La estrategia de desarrollo de Sierra Productiva está mediada por la apropiación de tecnologías simples y baratas que se convierten en herramientas imprescindibles para acompañar el proceso de construcción de autonomía comunitaria. En la dinámica de transferencia de tecnologías el protagonismo es de los(as) campesinos(as), por lo que se reafirma la centralidad de la participación comunitaria en una construcción colectiva y entre iguales. Se ha generado un proceso de articulación de las comunidades con la naturaleza y las tecnologías que se manifiesta, por ejemplo, en la apropiación de las prácticas agroecológicas para la producción, la preservación del suelo y la protección de la biodiversidad. También en el cuidado de las fuentes hídricas a través de soluciones como los baños secos o los biosistemas integrados para el tratamiento de aguas negras.

Las familias que participan de la propuesta de Sierra Productiva son propietarias de las tierras y tienen el control de las tecnologías, de la gestión del agua, de las semillas y los biofertilizantes, entre otros. El acceso a recursos ha sido un proceso liderado por la comunidad, lo cual garantiza el respeto de sus derechos. Toda la producción es agroecológica, balanceada y apropiada a la cultura andina. La búsqueda por controlar el modelo alimentario a escala local puede garantizar que las familias tengan acceso permanente a una alimentación adecuada y suficiente, y a canales de comercialización para la generación de ingresos.

Otro elemento destacable es el ámbito cultural que ha permitido fortalecer los vínculos comunitarios, y que presenta una manifestación histórica en las prácticas colectivas del ayni y la minka. Esta expresión cultural junto con los procesos históricos de organización sustenta un ejercicio político de solidaridad donde se busca el bienestar comunitario. Esta característica ha facilitado el ejercicio de educación horizontal permitiendo que las familias asuman compromisos con sus intereses y se involucren en un aprendizaje práctico y comunitario. De esta forma, se logra reducir las limitaciones simbólicas que pueden crearse por la jerarquización del conocimiento y así generar confianza, legitimidad, creatividad y motivación. Todo esto expande las libertades de las comunidades y retroalimenta los procesos de desarrollo.

Estas estrategias de viabilización de la pequeña agricultura familiar son enfáticas y demuestran que no basta con el acceso a tierras para crear condiciones de soberanía. La construcción de procesos de autonomía que se reflejen en el mejoramiento de las condiciones de vida de las comunidades exigen la garantía del acceso a recursos productivos, tecnologías, capacidades de organización y coherencia con el horizonte social que se persigue. Este último elemento puede ser foco de tensiones ante las iniciativas de escalamiento e institucionalización de la propuesta, por los intereses que intervienen en esos niveles de decisión. En particular, se quieren señalar las evidentes disputas y diferencias de proyectos de sociedad que se plantean entre la agricultura campesina y de la soberanía alimentaria con la agricultura empresarial de la dependencia.

\section{ANÁlisis ProspeCtivo}

Las características de los modos de vida campesina (Ploeg, 2014) pueden ser un marco de análisis que sustente la transmisión de principios de experiencias como la de Sierra Productiva a otros contextos con presencia de agricultura familiar empobrecida. En ese proceso la cultura es un elemento central para llevar en consideración, siendo fundamental identificar los valores y símbolos que fortalecen las prácticas de solidaridad en cada contexto. La ampliación del poder social en las comunidades puede contar con el apoyo de instituciones externas, pero ese acompañamiento requiere un cambio de paradigma. En este sentido, debe superarse la racionalidad productivista, la visión de corto plazo y la frecuente asunción de supremacía del conocimiento académico o especializado sobre otros tipos de saberes. Estos desafíos deben enmarcarse en un proceso de democratización del conocimiento y transformación de las relaciones sociales que atienda a la deuda con los sectores históricamente marginalizados. Un elemento de especial atención para trabajos futuros es analizar el potencial de esta experiencia para contrarrestar los procesos de éxodo rural. Los casi 24 años de permanencia de Sierra Productiva, el escalamiento a diversas regiones del Perú y la dinámica de innovación comunitaria y organizacional suponen aprendizajes significativos para enfrentar el vaciamiento de los campos e inclusive motivar procesos de retorno. Avanzar en estas consideraciones 
es un aporte destacable para el fortalecimiento de los proyectos de soberanía, dada la expresión generalizada del fenómeno del éxodo rural en América Latina.

\section{Agradecimientos}

A la CAPES por la beca de estudios. A Carlos Paredes y Haydée Romero por permitir aproximarnos a la propuesta de Sierra Productiva. A Nicanor, Marcelino, don Francisco y doña Trinidad por las atenciones y orientaciones. A los(a)s campesinos(as) que destinaron tiempo de su valioso día para las conversaciones.

\section{REFERENCIAS}

Altieri, M. A., \& Toledo, V. (2011). The agroecological revolution in Latin America: Rescuing nature, ensuring food sovereignty and empowering peasants. Journal of Peasant Studies, 8(3), 587-612.

Bajo Tierra. (2011). Pensar las autonomias. Alternativas de emancipación al capital y el estado [Prólogo]. México: Sísifo Ediciones, Bajo Tierra.

Barkin, D. (2002). El desarrollo autónomo: un camino a la sostenibilidad, en H. Alimonda (Ed.), Ecología política. Naturaleza, sociedad y utopia (pp. 169-202). Buenos Aires: CLACSO.

Barkin, D. y Lemus, B. (2015). Construyendo mundos pos-capitalistas. Cultura y representaciones sociales, 26-60.

Baschet, J. (2015). Adiós al capitalismo: autonomia, sociedad del buen vivir y multiplicidad de mundos. México: Ned ediciones.

Caporal, F. R. e Costabeber, J. A. (2004). Agroecologia: alguns conceitos e princípios. Mda/Saf/Dater-Iica, I, 24. http://doi.org/10.1590/S0103-20032005000100009

Carpio, J. (2002). Desarrollo local en los espacios rurales. Polis, 2.

Carpio, J. y Elizalde, A. (2009). Lo local: ámbito de contención de la globalización «perversa ». Polis, 22.

CIPAF (Centro de Investigación y Desarrollo Tecnológico para la Agricultura Familiar)-INTA (Instituto Nacional de Tecnología Agropecuaría). (2009). Energías renovables para el desarrollo rural. Buenos Aires: INTA.

Cotler, J. y Portocarrero, F. (1976). El campesinado, en J. M. Mar (Ed.), Hacienda, comunidad y campesinado en el Perú (p. 390). Lima: IEP ediciones.

Eguren, F. (2006). Reforma agraria y desarrollo rural en el Perú, en F. Eguren (Ed.), Reforma agraria y desarrollo rural en la región andina. Perú: CEPES.

Fernandes, B. M. (2017). Territorios y soberanía alimentaria. ReLaER, 2(3), 22-39.

Fernández-Baldor, Á. (2014). Technologies for freedom: una aproximación a los proyectos de cooperación de corte tecnológico desde el enfoque de capacidades. Valencia: Editorial Universitat Politècnica de València.

Francis, C. A., \& Wezel, A. (2015). Agroecology and agricultural change. International Encyclopedia of the Social y Behavioral Sciences, 1, 484-487.

Guber, R. (2011). La etnografía-método, campo y reflexividad. Buenos Aires: Siglo Veintiuno Editores.

Heinisch, C. (2013). Soberanía alimentaria: un análisis del concepto, en F. Hidalgo, P. Lacroix, y P. Román (Eds.), Comercialización y soberanía alimentaria (pp. 11-35). Quito: SIPAE.

Illich, I. (1978). La convivencialidad. México: Planeta.

Mariátegui, J. (1928/1986). 7 ensayos de interpretación de la realidad peruana (48a ed.). Lima: Amauta.

Martinez-Torres, M. E. y Rosset, P. (2014). Diálogo de saberes: la construcción colectiva de la soberanía alimentaria, en Soberanía alimentaria: Un diálogo crítico (pp. 147-159). País Vasco: EHNE, ETXALDE, ICAS. 
Matos-Mar, J. (2005). Desborde popular y crisis del Estado. Veinte años después. Lima: Fondo Editorial del Congreso del Perú.

Matos Mar, J. y Mejía, J. (1980). La reforma agraria en el Perú. Lima: IEP ediciones.

Max-Neef, M., Elizalde, A. y Hopenhayn, M. (2010). Desarrollo a escala bumana: una opción para el futuro-Segunda Parte. Desarrollo y necesidades humanas. Madrid: CF+S, Biblioteca.

Morante, F. (2007). Transferencia tecnológica de campesino a campesino: la experiencia de los yachachiq de la micro cuenca del Jabón Mayo, en R. Zielles (Ed.), Experiencias de transferencias de tecnologías con uso de energias renovables. São Paulo: IEE-USP/RITTAER.

Paredes, C. (2008). Los yachachiq: una experiencia de desarrollo inclusivo y sostenible. Leisa, revista de agroecología, 24(3), 39-41.

Pimbert, M. (2009). Transformando el conocimiento y las formas de saber. Hacia la soberanía alimentaria. Reclamando los sistemas alimentarios autónomos. London: IIED

Ploeg, J. D. Van Der. (2014). Dez qualidades da agricultura familiar. Agriculturas: experiências em agroecologia, $1,7-14$.

Portales, L. (2014). Los pobres como agentes de su desarrollo, la lucha contra la pobreza y la exclusión desde lo local. Revue Interventions économiques, 50.

Porto-Gonçalves, C. W. (2006). A geograficidade do social: uma contribuição para o debate metodológico para o estudo de conflitos e movimentos sociais na América Latina. Revista Eletrônica AGB-TL.

Porto-Gonçalves, C. W. (2009). De saberes y de territorios-diversidad y emancipación a partir de la experiencia latino-americana. Polis, 22.

Rénique, J. (2009). A revolução peruana. M. Lopes (Trad). São Paulo: Unesp.

Rivera-Ferre, M. G. (2008). Soberanía alimentaria: limitaciones y perspectivas, en Derecho a la alimentación y soberania alimentaria (pp. 105-119). España: Universidad de Córdoba.

Rosas, F. (2009). Breve historia general de los peruanos. Arequipa: El lector.

Rosset, P. M. (2016). La reforma agraria, la tierra y el territorio: evolución del pensamiento de La Vía Campesina. Mundo Agrario, 17(35).

Sampieri, R. H., Collado, C. F. y Lucio, P. B. (2010). Metodología de la investigación. Ciudad de México: McGraw-Hill.

Sevilla Guzmán, E. y Montiel, M. (2010). Agroecología y soberanía alimentaria: alternativas a la globalización agroalimentaria. Ph Cuadernos, 26, 316.

Thomas, H. (2011). Tecnologías sociales y ciudadanía socio-técnica. Notas para la construcción de la matriz material de un futuro viable. Revist@do Observatório do Movimento pela Tecnología Social da América Latina, 1(1), 1-22.

Toledo, V. (2009). Ecología política, sustentabilidad y poder social en Latinoamérica. ALAI, 445, 6-9.

Velarde, O. del C. (2006). Evaluación final del proyecto "Capacitación para Tecnificación y Energización del Agro de Pequeña Producción Campesina” (Informe final). Documentos internos IAA.

Villanueva, V. (1967). Hugo Blanco y la rebelión campesina. Lima: Juan Mejía Baca

Vivanco, M. F. y Bellatín, P. (2013). Innovación y participación para el desarrollo de la pequeña producción campesina: la experiencia del programa "Sierra Productiva”, en Á. Paz, M. P. Montoya y R. H. Asensio (Eds.), Escalando innovaciones rurales (pp. 235-256). Lima: IEP-IDRC-CRDI-FIDA.

\section{BY-NC-N}

